\title{
Nanostructured surface dental implants, a modern solution for the treatment of patients with chronic systemic diseases.
}

\author{
Mircea Suciu ${ }^{1}$, Dragoș Vladimir Budei ${ }^{2}$, Florentin Daniel Berneanu ${ }^{1}$ \\ ${ }^{1}$ George Emil Palade University of Medicine, Pharmacy, Science, and Technology of Târgu-Mureș, Romania \\ 2 Polytechnic University of Bucharest, Romania
}

\begin{abstract}
Introduction. In modern dentistry the oral rehabilitation of patients with the help of dental implants has a very high success rate. However, the problem is the situation of patients with serious chronic diseases in which the insertion of dental implants is problematic or dental procedures can complicate or aggravate the patients' disease.

Case report. We presented the case of a patient with associated chronic diseases who benefited from the insertion of implants with a nanostructured surface. The nanotube surface of the dental implant has been loaded with antiinflammatory drugs to promote healing and stimulate the process of osteogenesis.

Conclusions. Within the limitations of this study, we consider the therapy with avant-garde nanostructured dental implants to represent a viable treatment option for patients with a medical history with complicated chronic diseases. Keywords: oral rehabilitation, dental implants, chronic disease, nanostructured surface, ostegenesis.
\end{abstract}

\section{Introduction}

Oral rehabilitation of patients with dental implants, in the era of modern dentistry, is predictable and with a success rate of over $90 \%$ [1]. However, the literature indicates variable percentages of situations of loss of dental implant, among which we mention perimucositis, peri-implantitis, loss of osseointegration, poor oral hygiene, etc. [2,3]. In 2015 World Health Organization publishes in its report, the list of the most common diseases of the elderly that can influence the treatment with dental implants, putting in the first place cardiovascular disease (CVD), cancer, respiratory diseases, diabetes mellitus, cirrhosis of the liver, osteoarthritis, etc. [4]. Castellanos-Cosano et. al. (2019) addressed the relationship between dental implant loss and patient systemic disease and observed that there is a statistically significant risk of dental implant loss in patients with diabetes mellitus over 70 years of age and in patients with CVD between $61-70$ years $(p<0.001)$ [5]. That is why these chronic systemic diseases and the multiple possibilities of degradation of the biological support or mechanical trauma, which lead to the loss of the dental implant, have always raised problems for specialists in carrying out an individualized treatment, modeled on the specific basic medical conditions [6].

There were over 220 types of dental implants in the early 2000s with an estimated number of over 2000 designs [7]. In order to improve the survival rate of a dental implant, the specialists defined the three important elements that characterize an implant, its geometry, the material and the way of making the surface [8].

If we talk about the geometry of the cylindrical or conical implant, there are studies that consider it a little important, but also vice versa, being considered important. Instead, everyone considers that the most important aspect for obtaining primary stability is the torq [9].

If we approach the material, we can say that pure commercial titanium ( $\mathrm{Ti} 12$ ) showed better cell viability than Ti-6Al-4V alloys (Ti 5, $\mathrm{Ti}$ 23), although a significant decrease in cytotoxicity due to $\mathrm{TiO} 2$ formation was observed in both types of materials [10]. Some recent studies have questioned the cytotoxicity deficiencies of Ti 5 and Ti 23, which contains vanadium and aluminum, suggesting that in vitro cytotoxicity is different from in vivo, due to different ionic concentrations [11]. Budei et al. (2021) showed that many implant manufacturers prefer Grade 4 pure titanium (Ti 
CP4) as their material due to the lack of homogenity in different alloys [12].

If we talk about the surface of the dental implant, a simple search in PubMed (5 years ago, free full text) showed us 1240 articles that debate or propose an efficient surface of the dental implant. If we did the same search for nanotube dental implants we found only 58 results. There have been authors who have used nanotubes to increase the osseointegration of implants with the help of variants of human proteins, collagen or even the in vitro modification of the surface structure [13-15]. Yang et al. (2020) demonstrated through an in vitro study that changing the surface of the dental implant by making nanotubes attenuates the inhibition of osteogenesis induced by diabetes mellitus, this structure giving the dental implant a favorable surface for the diabetic patient [16]. Pathak et al (2019) managed to successfully produce TiO2 nanotubes by combustion synthesis on the surface of the dental implant [17]. The study by Camargo et al. (2021) showed that $\mathrm{Ti}$ nanotubes can promote the proliferation of osteoblasts and reduce the adhesion of bacterial biofilm to the surface of the dental implant, which is important to achieve good osseointegration [18].

\section{Case report}

We present the case of a 61-year-old male patient, working in a toxic (chemical) environment, who presented to the clinic for a complex oral rehabilitation treatment, with the express request to avoid the application of complete dentures. The general medical history showed the existence of an acute myocardial infarction 13 years ago and a coronary stent applied 1 year ago. The patient does not have high blood pressure and is a non-smoker. The patient's current medication was instituted by the cardiologist and is as follows: Plavocorin 75 $\mathrm{mg} /$ day, Nebivolol $5 \mathrm{mg} /$ day and Atorvastatin $10 \mathrm{mg} /$ day.

From the point of view of the dental situation, the patient was completely edentulous in the jaw, being the bearer of a complete deficient denture for 6 years. In the lower jaw, the patient had Kennedy class III/2 edentation. The restoration was done with an unsuitable metal-ceramic bridge (figure 1).

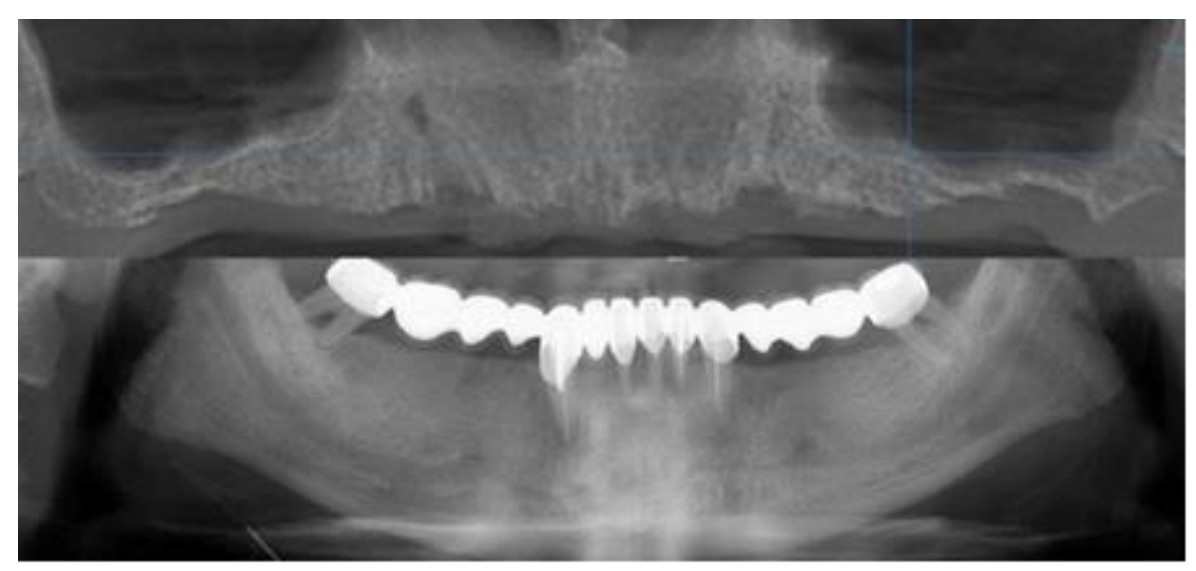

Figure 1. Initial status

In order to clarify the diagnoses and to establish a dental treatment plan, the following paraclinical investigations were requested: cardiological consultation with the attending physician, complete blood tests and CBCT investigation. The cardiologist confirmed the general medical diagnoses and maintained the current treatment and made the recommendation that in case of implant insertion the anticoagulant medication be interrupted one day before implantation and resumed after two days. Broad-spectrum antibiotics have also been associated for 7 days. Laboratory analyzes showed normal values for all parameters except: monocytes 0.67 (reference range 0.1-0.6 $10^{\wedge} 3 / \mu \mathrm{L}$ ), basophils 0.14 (reference range $0.01-0.0810^{\wedge} 3 / \mu \mathrm{L}$ ), mean erythrocyte volume 100.50 (reference range $78-95 \mathrm{fL}$ ), platelet distribution width 22.22 (reference range 10-22 fL), Quik time 14 
(reference range 9.8-12.1 seconds), Activated Partial Thromboplastin Time (APTT) 37.30 (reference range 23-31.9 seconds) and APTT Ratio is 1.52 (reference range $0.9-1.1$ seconds). The CBCT evaluation confirmed the previously made diagnoses and revealed a D3 bone density in the jaw and mandible, but with a minimum width of the edentulous maxillary ridge between 4.65 and $4.8 \mathrm{~mm}$ in quadrant 1 and 5.25-5.6 in quadrant 2. In mandible width the crest was on average $5.52 \mathrm{~mm}$. Under these conditions, the patient was proposed to simulate the implant treatment and then to perform the Dentix Nano ${ }^{\circledR}$ implant treatment, a treatment plan that was accepted. The decision to use this type of nanostructured implant was based on three important arguments: the patient's medical condition, the fact that the implant has a surface with nanotubes and the possibility of loading the nanotubes with antibiotics or antiinflammatory as needed drugs, delivered in-situ for a long period of time. Implant treatment was performed by inserting five implants in the jaw and six implants in the mandible as seen in figure 2, and the decision to hydrophilize the nanotubes was in favor of dexamethasone (DEX) which reduced local inflammation, pain and postoperative edema. Together with the broad-spectrum antibiotic administered before and after implantation, they favored a better cure later. Hydrophilization of nanotubes on the surface of dental implants was performed in the office by immersing the implant in sterile dexamethasone solution and ultrasonic vibration of the implant (figure $3 \mathrm{~A}, \mathrm{~B}$ ).

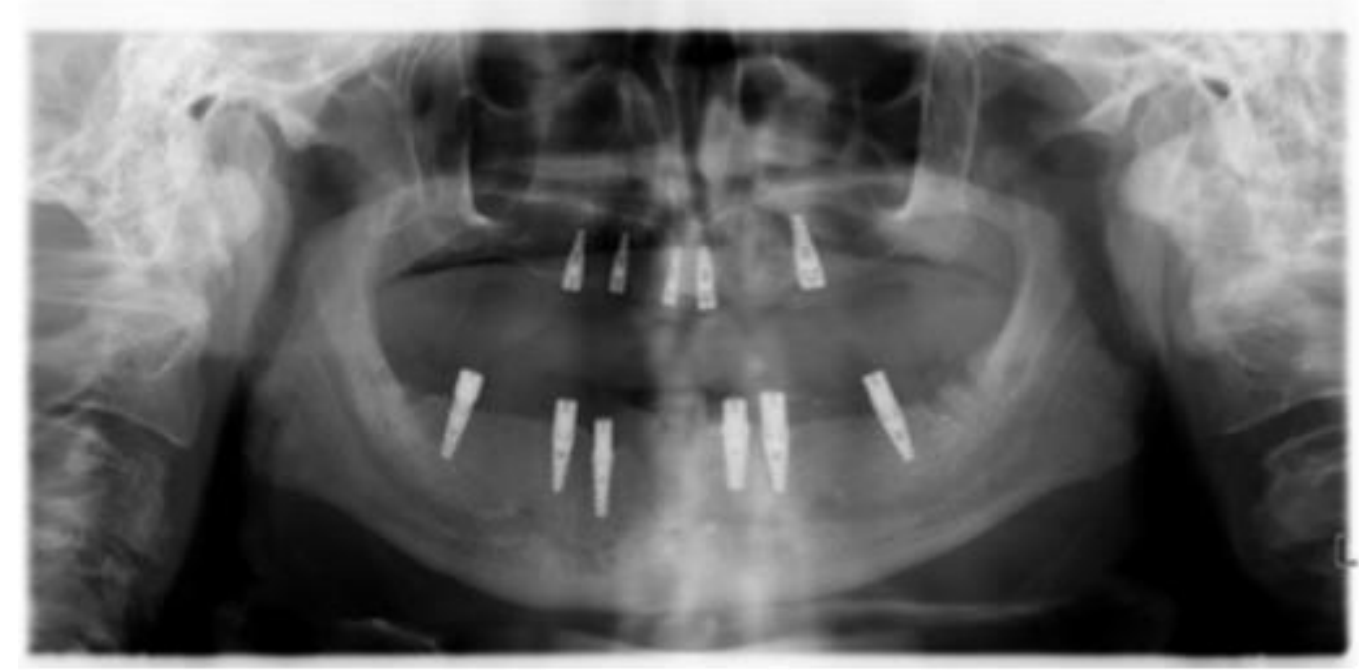

Figure 2. Maxilar and mandibular implants placement
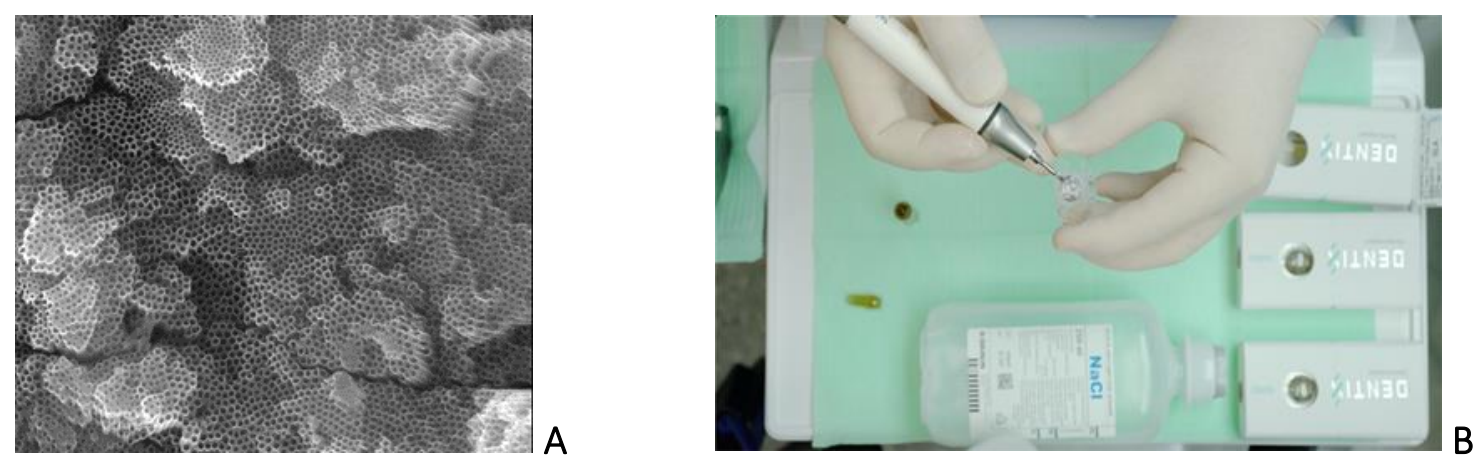

Figure 3. A-Nanostructured surface with a layer of Titania nano-tubes on a Dentix Nano dental implant, B-Loading nano-tubes with Dexamethasone

The temporary prostheses for the jaw were made by the foliation of the old complete dentures in the area of dental implants insertion and the application of resilient lining material, and a temporary milled acrylic work was made for the mandible (figure 4). 


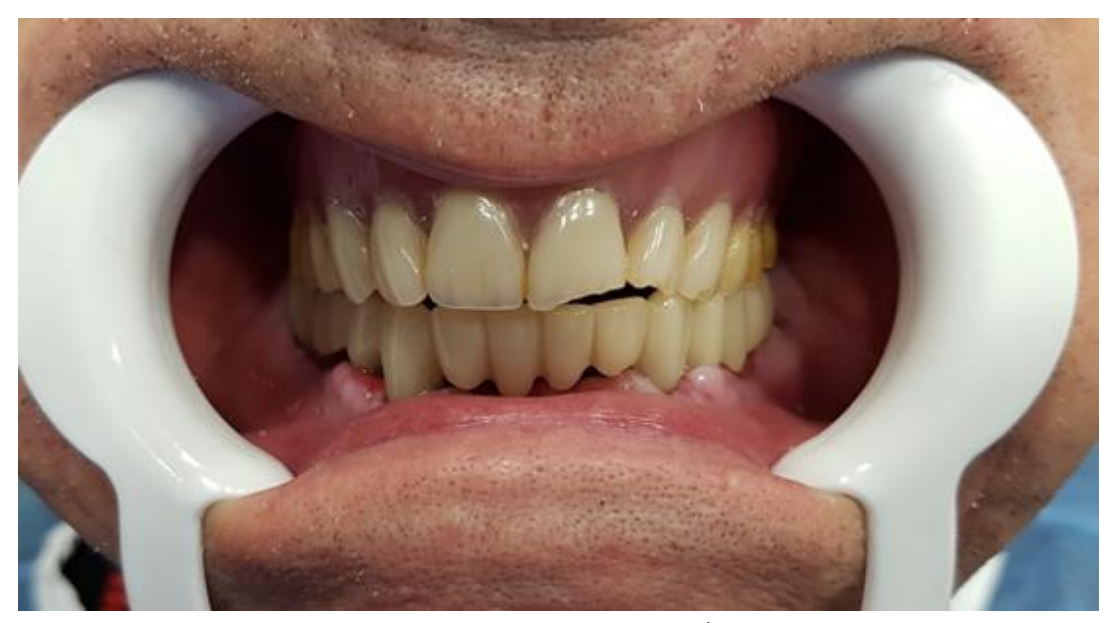

Figure 4. Temporary prosthesis

Five months after insertion due to the mechanical forces transmitted by the prosthesis on the implant area from position 2.5 , it was pushed into the maxillary sinus (figure 5). The patient did not report any allegations about this. The implant was removed from the sinus and another similar implant was applied in position 2.4.

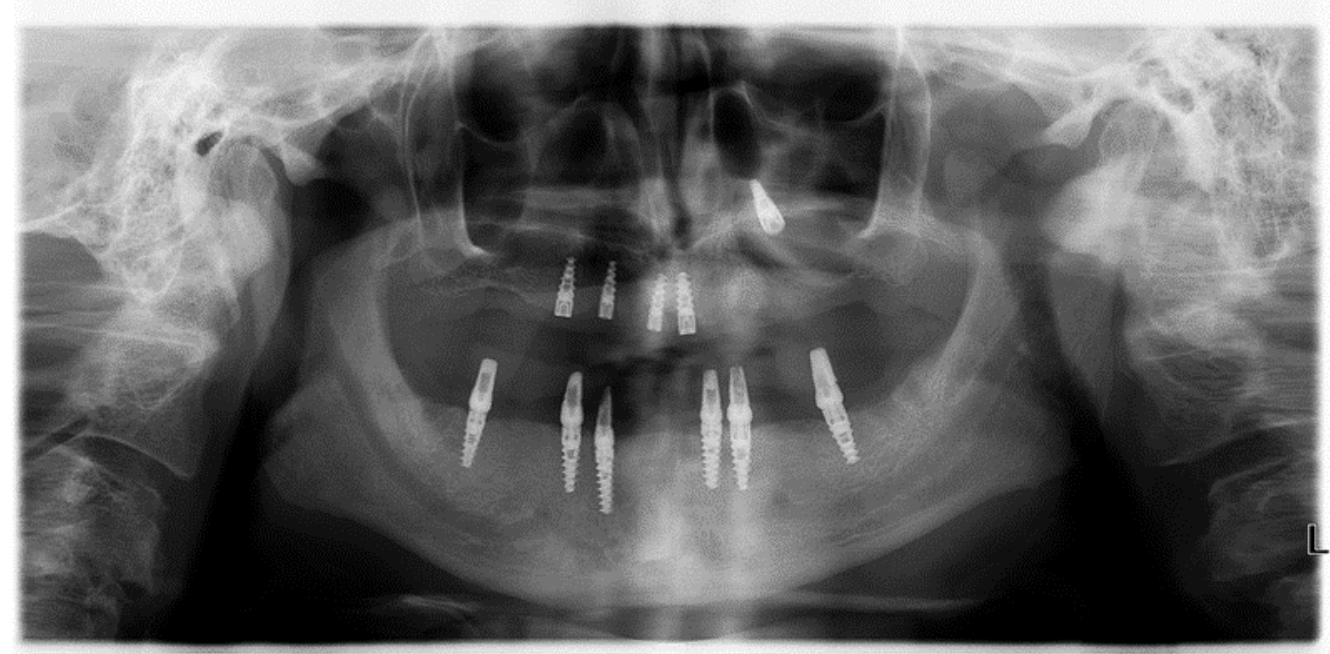

Figure 5. 2.5 Dental implant migrated to maxillary sinus

After the osseointegration period, the final prosthetic treatment was performed. A complete overdenture anchored on implants with a system of connecting bars and palatal vault not covered by the prosthesis was made at the maxilla, and a cemented metal-ceramic bridge with implant support was made at the mandible (figure 6). 


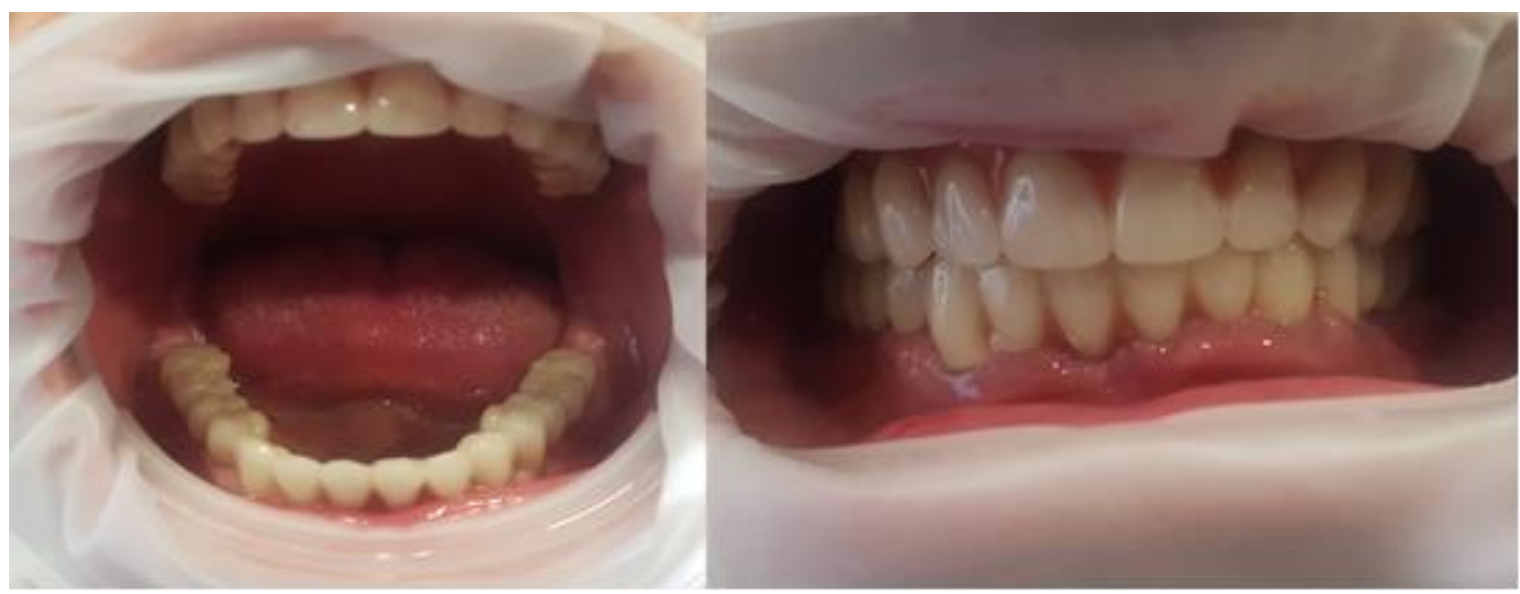

Figure 6. Final status

\section{Discussions}

All Titanium based dental implants present a Titania (TiO2) nano-layer on the surface due to the specific behaviour of Titanium in contact with Oxygen. In all dental implants with a SLA surface treatment, this nanolayer (few nanometers thick) is amorphous, or nonstructured. Live tissue receiving a Titanium based implant, will directly interfere with this Titania nano-layer. The real molecular chemotaxis mechanism subsequent to osseointegration, is trigged by the Titania. One of the reasons behind choosing to structure this nano-layer of Titania into nanotubes arrays, was to empower this chemotaxis mechanism. At the size of 40-60um inner diameter, 60-80um outer diameter and 100$200 \mathrm{um}$ in height, the surface of Titania exposed to the live tisuse is more than 6 times higher than on the same size SLA implant[12].

Another reason was that this Titania nanotubes arrays may be used as drug delivery systems (DDS), once they have been loaded with appropriate drugs $[19,20]$. This opens the perspective to customize the implant treatment to any patient and to make it possible for those with major chronic diseases.

Special production procedures of this arrays, allow to control the diameter (inner and outer) and the height of the nanotubes and hence, to control the drug delivery through the elution mechanism.

The healing process in the oral cavity goes through the same stages every time. One of these stages is the inflammatory reaction produced by cytokines. The role of this stage is to remove tissue debris, necrotic tissue and to some extent microorganisms [21]. It was decided to use DEX to hydrophilize the surface of the dental implant for two reasons. The first was that these implants had to be hydrophilic to ensure osseointegration, and the second was the desire to modulate different phases of the healing process [22]. DEX, a synthetic glucocorticoid, is known and used clinically as an anti-inflammatory drug. Previous studies have reported that DEX can induce osteoporosis or even pathological fractures [23], while DEX in vitro promotes osteoblast differentiation and bone mineralization [24-26]. In fact, DEX has been commonly used to induce mesenchymal stem cell differentiation and is a key component in osteogenic differentiation. However, different effects of DEX on stem cells and undifferentiated osteoblasts have been reported [27]. Specifically, a low concentration of DEX increases the activity of stem cells and promotes differentiation in the healing process, and high concentrations and long-term treatments negatively influence this process [28].

Yang (2021) in a retrospective study mentioned the possible causes of dental implant failure shortly after insertion, incriminating the lack of osseointegration due to dehiscence and wound infection, osteonecrosis due to improper torque, bone perforations during insertion, loading premature implantation or application of excessive force, or periodontitis of adjacent teeth [29].

Do (2020) showed in a literature review that many factors may be involved that cause late 
dental implant failure. Common risk factors related to late dental implant failure could be classified into three groups, patient history (radiotherapy, bruxism, periodontitis, and early implant loss), clinical parameters (grade 4 bone and implant placed in a posterior location), and decisions made by the doctor (low initial stability, more than one implant placed during surgery or using a sustained implant overdenture with cone connection) [30]. Taking into account the presented studies, we considered that the early loss of the implant in quadrant 2 was due to the application of inadequate forces, probably transmitted by the imperceptible balance of complete dentures applied to the patient in the temporary prosthesis stage.

After completing these steps, the patient was rehabilitated with the final prosthetic parts according to standard procedures. An overdenture with implant support on the connecting bar was performed on the maxilla. A dental bridge anchored to dental implants was not an option due to the size of the dental implants in the first quadrant of the maxilla. A dental bridge support on six dental implants was applied to the mandible.

\section{Conclusions}

Careful study of the patient's medical history and correlation of dental treatment options with the patient's chronic diseases made it possible to take therapeutic measures that favored the healing processes after the insertion of dental implants. A great help was the one offered by the Dentix Nano implants, which allowed the placement of drugs and their gradual release, at the place of the insertion of the dental implants. Within the limitations of this study, subject to the short time of monitoring the case (20 months), we consider the therapy with this type of nanostructured dental implants represent a viable treatment option.

Conflict of interest: Mr. Mircea Suciu and Mr. Florentin Daniel Berneanu report no conflict of interest. Mr. Dragos Budei is a shareholder of Dentix Millennium and the inventor of the nano-structuring method applied on described medical devices.

\section{Acknowledgments}

This research was supported by Dentix Millennium SRL through the project "Dental implants surface functionalization for improved osseo- integration - an innovative method" ("Metoda inovativă pentru funcționalizarea suprafețelor implanturilor dentare cu scopul îmbunătățirii osteointegrării”') code MySMIS 104809, contract no. ANCSI 73/8.09.2016.

\section{References}

1. Hickin MP, Shariff JA, Jennette PJ, Finkelstein J, Papapanou PN. Incidence and Determinants of Dental Implant Failure: A Review of Electronic Health Records in a U.S. Dental School. J Dent Educ. 2017;81:1233-42.

2. Derks J, Tomasi C. Peri-implant health and disease: a systematic review of current epidemiology. J Clin Periodontol. 2015;42:S158-71.

3. Insua A, Monje A, Wang HL, Miron RJ. Basis of bone metabolism around dental implants during osseointegration and peri-implant bone loss. J Biomed Mater Res A. 2017;105(7):2075-2089.

4. World Health Organization. World report on ageing and health. Geneva, Switzerland: WHO Press; 2015.

5. Castellanos-Cosano L, Rodriguez-Perez A, Spinato $S$, et al. Descriptive retrospective study analyzing relevant factors related to dental implant failure. Med. Oral Patol. Oral Cir. Bucal. 2019;24:e726e738.

6. Horikawa $T$, Odatsu $T$, Itoh $T$, et al. Retrospective cohort study of rough-surface titanium implants with at least 25 years' function. Int. J. Implant Dent. 2017;3:42.

7. Jokstad A, Braegger U, Brunski JB, Carr AB, Naert I, Wennerberg A. Quality of dental implants. Int Dent J. 2003;53(6 Suppl 2):409-43.

8. Romanos G, Ciornei G, Jucan A, Malmstrom H, Gupta B. Clinical Implant Dentistry and Related Research. 2014;16(1):89-95.

9. Staedt H, Palarie V, Staedt A, et al. Primary Stability of Cylindrical and Conical Dental Implants in Relation to Insertion Torque-A Comparative Ex Vivo Evaluation. Implant Dent. 2017;26(2):250255.

10. Chandar S, Kotian R, Madhyastha P, Kabekkodu SP, Rao P. In vitro evaluation of cytotoxicity and corrosion behavior of commercially pure titanium and Ti-6Al-4V alloy for dental implants. J Indian Prosthodont Soc. 2017;17(1):35-40.

11. Willis J, Li S, Crean SJ, Barrak FN. Is titanium alloy Ti-6Al-4 $\mathrm{V}$ cytotoxic to gingival fibroblasts-A systematic review. Clin Exp Dent Res. 2021;1-8. 
12. Budei D, Vaireanu D, Prepelita P, Popescu-Pelin G, Mincu M, Ciobotaru I. A comparative morphological study of titanium dioxide surface layer dental implants. Open Chemistry.2021;19(1):189-198.

13. Lee JK, Choi DS, Jang I, Choi WY. Improved osseointegration of dental titanium implants by $\mathrm{TiO} 2$ nanotube arrays with recombinant human bone morphogenetic protein-2: a pilot in vivo study. Int J Nanomedicine.2015;10:1145-1154.

14. Yeo IL. Modifications of Dental Implant Surfaces at the Micro- and Nano-Level for Enhanced Osseointegration.Materials(Basel).2019;13(1):89.

15. Zhang Y, Hu L, Lin M, Cao S, Feng Y, Sun S. RhBMP2-Loaded PLGA/Titanium Nanotube Delivery System Synergistically Enhances Osseointegration. ACS Omega.2021;6(25):16364-16372.

16. Yang J, Zhang $H$, Chan SM, et al. TiO2 Nanotubes Alleviate Diabetes-Induced Osteogenetic Inhibition. Int J Nanomedicine.2020;15:35233537.

17. Pathak TK, Kroon RE, Craciun V, Popa M, Chifiriuc $\mathrm{MC}$, Swart HC. Influence of Ag, Au and Pd noble metals doping on structural, optical and antimicrobial properties of zinc oxide and titanium dioxide nanomaterials. Heliyon.2019;5(3):e01333.

18. Camargo SEA, Xia X, Fares C, et al. Nanostructured Surfaces to Promote Osteoblast Proliferation and Minimize Bacterial Adhesion on Titanium. Materials(Basel). 2021;14:4357.

19. Aw MS, Addai-Mensah J, Losic D. A multi-drug delivery system with sequential release using titania nanotube arrays. Chem. Commun. 2012;48:3348-3350.

20. Kunrath MF, Penha N, Ng JC. Anodization as a promising surface treatment for drug delivery implants and a non-cytotoxic process for surface alteration: a pilot study. J Osseointegr. 2020;12(1):XXXX.

21. Politis C, Schoenaers J, Jacobs R, Agbaje JO. Wound Healing Problems in the Mouth. Front Physiol. 2016;7:507.

\section{Corresponding author:}

Budei Dragoș Vladimir

Polytechnic University of Bucharest, Splaiul Independenței 313, Bucharest 060042, Romania

Email: budei.dragos@dentixmillennium.ro
22. Abreu AM, Douglas de Oliveira DW, Marinho SA, Lima NL, de Miranda JL, Verli FD. Effect of topical application of different substances on fibroplasia in cutaneous surgical wounds. ISRN Dermatol. 2012;2012:282973.

23. Sambrook PN. Glucocorticoid-induced osteoporosis. International Journal of Rheumatic Diseases. 2008;11(4):381-385.

24. Cheng SL, Yang JW, Rifas L, Zhang SF, Avioli LV. Differentiation of human bone marrow osteogenic stromal cells in vitro: induction of the osteoblast phenotype by dexamethasone. Endocrinology. 1994;134(1):277-286.

25. Eijken M, Koedam M, van Driel M, Buurman CJ, Pols HAP, van Leeuwen JPTM. The essential role of glucocorticoids for proper human osteoblast differentiation and matrix mineralization. Molecular and Cellular Endocrinology. 2006;248(12):87-93.

26. Igarashi M, Kamiya N, Hasegawa M, Kasuya $T$, Takahashi $T$, Takagi $M$. Inductive effects of dexamethasone on the gene expression of Cbfa1, Osterix and bone matrix proteins during differentiation of cultured primary rat osteoblasts. Journal of Molecular Histology. 2004;35(1):3-10.

27. Wang $H$, Pang B, Li Y, Zhu D, Pang T, Liu Y. Dexamethasone has variable effects on mesenchymal stromal cells. Cytotherapy. 2012;14(4):423-430.

28. Zhao M, Li P, Xu H, et al. Dexamethasone-Activated MSCs Release MVs for Stimulating Osteogenic Response. Stem Cells Int. 2018;2018:7231739.

29. Yang $Y, H u H$, Zeng $M$, et al. The survival rates and risk factors of implants in the early stage: a retrospective study. BMC Oral Health. 2021;21(1):293.

30. Do TA, Le HS, Shen YW, Huang HL, Fuh LJ. Risk Factors related to Late Failure of Dental Implant-A Systematic Review of Recent Studies. Int J Environ Res Public Health. 2020;17(11):3931. 